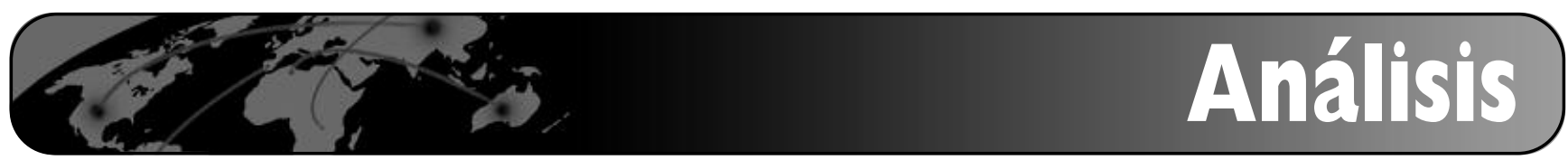

\title{
Análisis y propuestas sobre la suscripción de revistas del sistema bibliotecario de la Universidad Nacional Autónoma de México
}

\author{
Por María Esther Ramírez Godoy y Alma Silvia Díaz Escoto
}

\begin{abstract}
Resumen: Dentro de la amplia gama de recursos de información que ofrece la Dirección General de Bibliotecas (DGB) de la Universidad Nacional Autónoma de México (UNAM), las revistas ocupan un lugar relevante. El trabajo se inicia con un análisis financiero de las revistas impresas y electrónicas adquiridas por la DGB en el período 1999-2004, posteriormente

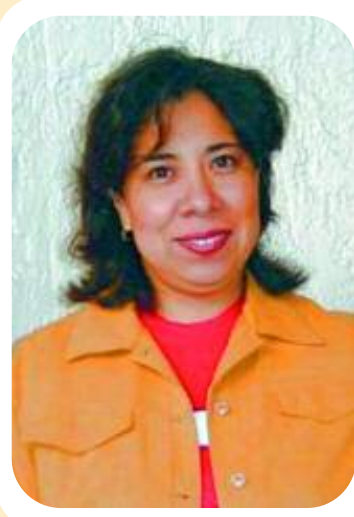
María Esther Ramírez Godoy, especialista
en recursos electrónicos, es administrado-
ra de recursos electrónicos de Ebsco,
Emerald, Swets y Wiley contratados por
la Universidad Nacional Autónoma de
México.
Alma Silvia Díaz Escoto, historiadora. Es-
pecialista en recursos electrónicos en
ciencias sociales y humanidades. Partici-
pa en el Seminario de Información del
Colegio Académico de la DGB y en el
proyecto Desarrollo de Habilidades Infor-
mativas de la DGB, presencial y en línea. hace referencia a los proyectos que se han generado para afrontar la crisis de las revistas, a fin de poder mantener en forma eficiente el servicio que presta el sistema bibliotecario de la UNAM. De igual forma, se describen los compromisos y actuaciones de las bibliotecas que llevan a cabo la adquisición cooperativa de recursos de información. El elemento fundamental de estos recursos son las revistas, independientemente del formato en que se adquieran, y el propósito principal es compartir la información entre las 142 bibliotecas que conforman la red universitaria.
\end{abstract}

Palabras clave: Revista impresa, Revista electrónica, Financiación, Consorcio, Universidad Nacional Autónoma de México, UNAM.

Title: Analysis and proposals concerning electronic journal subscriptions at the library system of the National Autonomous University of Mexico

\begin{abstract}
Journals are among the most prominent resources among the great range offered on the Web site of General Direction of Libraries (DGB) of the National Autonomous University of Mexico (UNAM), which serves to support the information requirements of UNAM's community. This work begins with a financial analysis of both the print and electronic journals purchased by the DGB from 1999 to 2004. It then presents projects implemented by the UNAM's library system for responding to the journal crisis in order to maintain an efficient service. A description follows of the agreements and activities undertaken by the libraries that carry out cooperative purchasing of information resources. The essential element among these resources are the journals, regardless of the format they are acquired in, and the main purpose is for sharing information among the 142 libraries that make up the UNAM library network.
\end{abstract}

Keywords: Print journals, Electronic journals, Financing, Consortia, Information resources, National Autonomous University of Mexico, UNAM.

Ramírez Godoy, María Esther; Díaz Escoto, Alma Silvia. «Análisis y propuestas sobre la suscripción de revistas del sistema bibliotecario de la Universidad Nacional Autónoma de México». En: El profesional de la información, 2006, julio-agosto, v. 15, n. 4, pp. 290-296.

COMO ES BIEN SABIDO en el mundo de la información, las revistas científicas son el medio fundamental para la investigación, docencia y difusión de la cultura. Por lo mismo, para las bibliotecas universitarias es una prioridad contar con acervos completos y actualizados de revistas que cubran las necesidades de información de sus comunidades.
Sin embargo, el progresivo desfase que se produce entre las aportaciones de la Universidad Nacional Autónoma de México (UNAM) a la Dirección General de Bibliotecas $(D G B)$ y los costes de suscrip- 


\section{baratz}

\section{En primera línea en}

Sistemas de Información y Gestión del Conocimiento
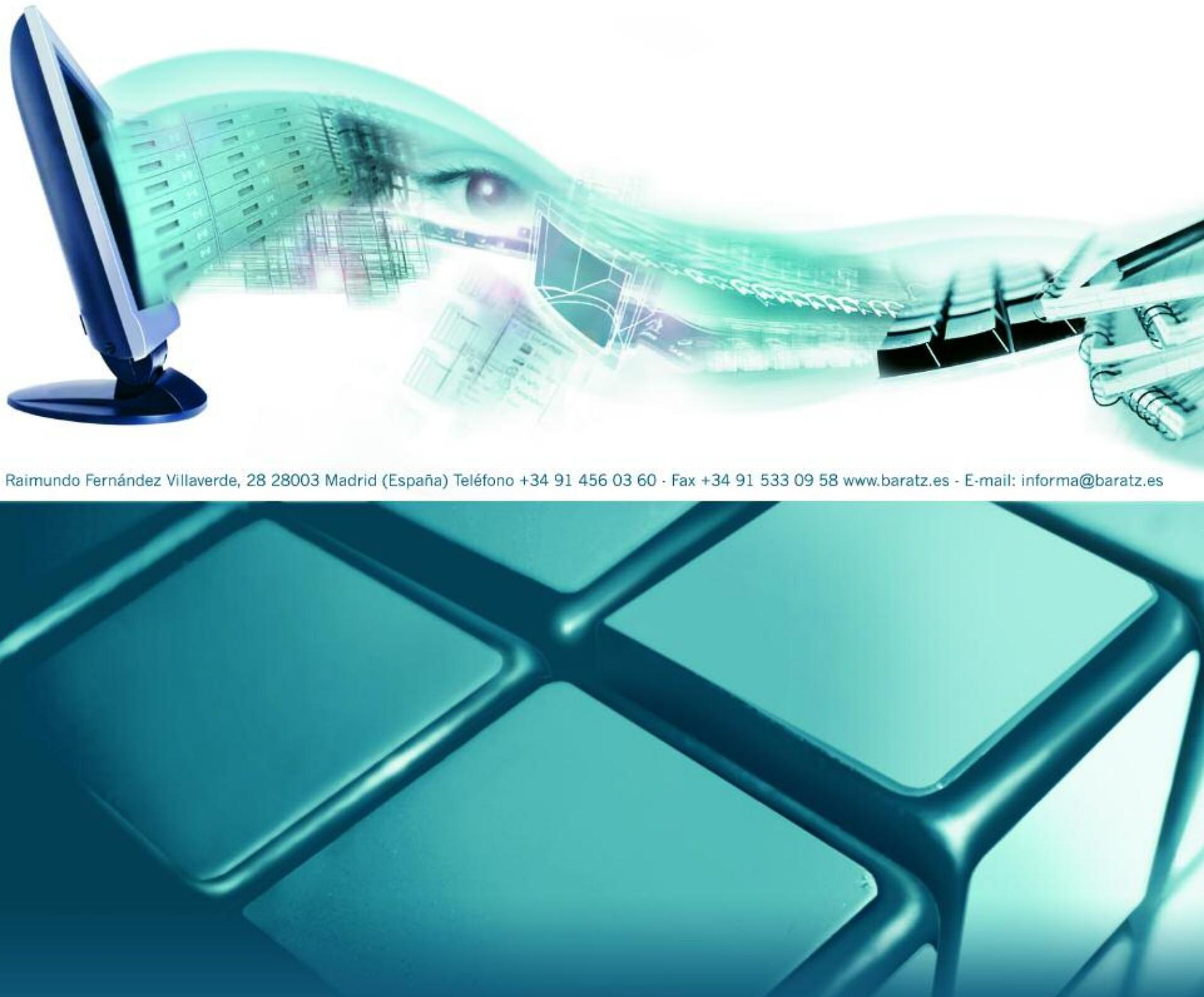

(2) Soluciones para bibliotecas: Absys, absys NET, Absys

(9) Gestión documental y del conocimiento: BKM, Baratz Windows, BRSCGI

(2) Soluciones para archivos: Albalá

(2) Servicios de Catalogación Retrospectiva

(9) Edición en CD-Rom

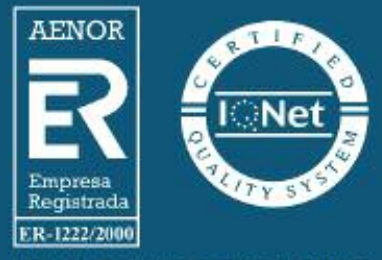




\begin{tabular}{|c|c|c|c|c|c|c|}
\hline Año & $\begin{array}{c}\text { Tipo de } \\
\text { cambio } \\
\text { US\$/Peso } \\
\text { mexicano }\end{array}$ & $\begin{array}{c}\text { Inversión } \\
\text { total } \\
\text { (US\$) }\end{array}$ & $\begin{array}{c}\text { Inversión en } \\
\text { suscripciones } \\
\text { en papel } \\
\text { (US\$) }\end{array}$ & $\begin{array}{c}\text { Porcentaje } \\
\text { respecto al } \\
\text { total (US\$) }\end{array}$ & $\begin{array}{c}\text { Inversión en } \\
\text { recursos } \\
\text { electrónicos } \\
\text { (US\$) }\end{array}$ & $\begin{array}{c}\text { Porcentaje } \\
\text { respecto al } \\
\text { total (US\$) }\end{array}$ \\
\hline 1999 & 10,23 & $10.173,60$ & $8.803,68$ & $82,91 \%$ & $1.369,92$ & $17,09 \%$ \\
\hline 2000 & 9,58 & $11.983,30$ & $9.751,27$ & $76,13 \%$ & $2.232,02$ & $23,87 \%$ \\
\hline 2001 & 9,51 & $12.133,41$ & $9.411,48$ & $73,57 \%$ & $2.721,92$ & $26,43 \%$ \\
\hline 2002 & 9,38 & $12.854,58$ & $10.048,43$ & $74,28 \%$ & $2.806,15$ & $25,72 \%$ \\
\hline 2003 & 10,52 & $11.484,57$ & $8.903,69$ & $73,49 \%$ & $2.580,87$ & $26,51 \%$ \\
\hline 2004 & 11,32 & $11.925,79$ & $8.657,24$ & $72,60 \%$ & $3.268,55$ & $27,40 \%$ \\
\hline
\end{tabular}

ción y adquisición de revistas, han obligado a las bibliotecas a racionar la insuficiencia de recursos, a fin de elegir cuidadosamente aquellos títulos que deben mantenerse y seleccionar de manera inteligente los títulos nuevos que es necesario adquirir, de tal forma que se logre integrar colecciones de revistas que puedan ser utilizadas y sustentadas de manera conjunta por un grupo de bibliotecas.

\section{«Cabe mencionar que en un principio el acceso a las revistas electrónicas era gratuito»}

Es importante destacar que en la medida en que las nuevas tecnologías han permitido acceder a los textos completos de numerosos títulos en forma digital, se ha procurado que la comunidad universitaria comparta los recursos electrónicos suscritos. La cancelación de cualquier suscripción duplicada impresa se compensa con la compra de nuevos títulos solicitados.

En un principio el acceso a las revistas electrónicas era gratis; más tarde, los mismos editores ofrecían el acceso electrónico gratis pero con la suscripción en papel. A partir del año 2000 aparecen numerosos modelos de tarifas y ese uso gratuito es casi inexistente.

La crisis generada por la desproporción entre el reducido incremento de presupuestos, y el aumento en el coste de las revistas cientí- ficas, además de la necesidad de adquirir recursos electrónicos emergentes, ha afectado a bibliotecas universitarias de todo el mundo. Como ejemplo está el caso de la Universidad del País Vasco, que ha tenido que suspender la suscripción de 496 revistas científicas ${ }^{1}$.

En este contexto, la intención de este artículo es dar a conocer la manera en que el sistema bibliotecario de la UNAM está afrontando este problema, así como expresar algunas sugerencias de planificación para aplicarse en el futuro inmediato.

De esta forma, en el año 1999 la $U N A M$ otorgó a la $D G B$ la cantidad de 10.173 US\$ para invertirla en recursos informativos, que se distribuyeron de la siguiente manera:

-8.804 US\$ para suscripciones impresas $(82,9 \%$ de la inversión total).

-1.369 US\$, para recursos electrónicos $(17,1 \% \text { restante })^{2}$.

En el año 2004 la inversión de la $U N A M$, a través de la $D G B$, fue de 11.926 US\$:

-8.657 US\$ para suscripciones impresas $(72,6 \%)$.

-3.269 US\$ para recursos electrónicos $(27,4 \%)^{3}$.

La tabla 1 nos muestra la evolución y la inversión en la adquisición de los títulos en formato impreso y electrónico en el período 19992004.
Como puede observarse, se ha incrementado la proporción gastada en revistas electrónicas mientras disminuye la inversión en las impresas. Hubo un leve aumento de 1.752 US\$ en la inversión total del presupuesto asignado a los recursos informativos en el período 19992004. Tomando los datos correspondientes al año 1999 y los de 2004, destaca la disminución de $10,3 \%$ en los gastos de suscripción a revistas impresas, mientras que aumentó el 10,3\% la inversión en revistas electrónicas. El incremento del $29,7 \%$ que ha recibido la $D G B$ para la adquisición de revistas científicas en esa etapa no es proporcional al que en estos años han tenido las revistas $(35 \%)^{4}$. Además, el total del aumento recibido se ha invertido en revistas electrónicas, lo que ha significado tener que suscribir menos títulos en papel y no poder adquirir nuevas publicaciones que la comunidad requiere; por otra parte, tampoco se han podido adquirir todos los títulos en formato electrónico que se necesitan.

\section{"Los editores y proveedores más importantes en el mundo de la información mantienen su línea en políticas de edición y distribución, con \\ precios en constante incremento»}

Para tener una visión más clara de estos cambios en revistas impre- 
sas y electrónicas, se presenta a continuación el gráfico de la figura $1^{5}$.

A pesar del leve aumento de los presupuestos destinados a la adquisición de recursos de información en los últimos cinco años, los datos estadísticos ${ }^{6}$ dejan claro que en las bibliotecas universitarias de la UNAM también está afectando la llamada crisis de las revistas, problemática estudiada desde hace más de dos décadas por profesionales de la información, tanto nacionales como extranjeros ${ }^{7}$.

En cuanto al tema de las suscripciones de revistas, las aportaciones emiten un dato de sumo interés: su consumo en diversos organismos públicos y privados es siempre mayor al presupuesto asignado, algo que está sucediendo año tras año, hasta la actualidad ${ }^{8}$. En la década de los 90, cuando se presenta la transición de las revistas en papel a otros formatos y formas de acceso, se plantea la necesidad de conservar las suscripciones impresas, además del formato electrónico, a sugerencia del usuario y a conveniencia de las propias bibliotecas. Esta acción tiene como consecuencia que las bibliotecas paguen costes adicionales, porcentajes que dependerán de los modelos de precios que establezcan los editores y proveedores de información ${ }^{9}$.

Debido al hecho del menor crecimiento de los presupuestos ${ }^{10}$ nos hemos visto obligados a realizar cancelaciones de títulos (tabla 2). Del año 1999 sólo se tienen datos inciertos ya que hubo huelga en la UNAM.

La $D G B$ tiene como política interna suscribir solamente títulos

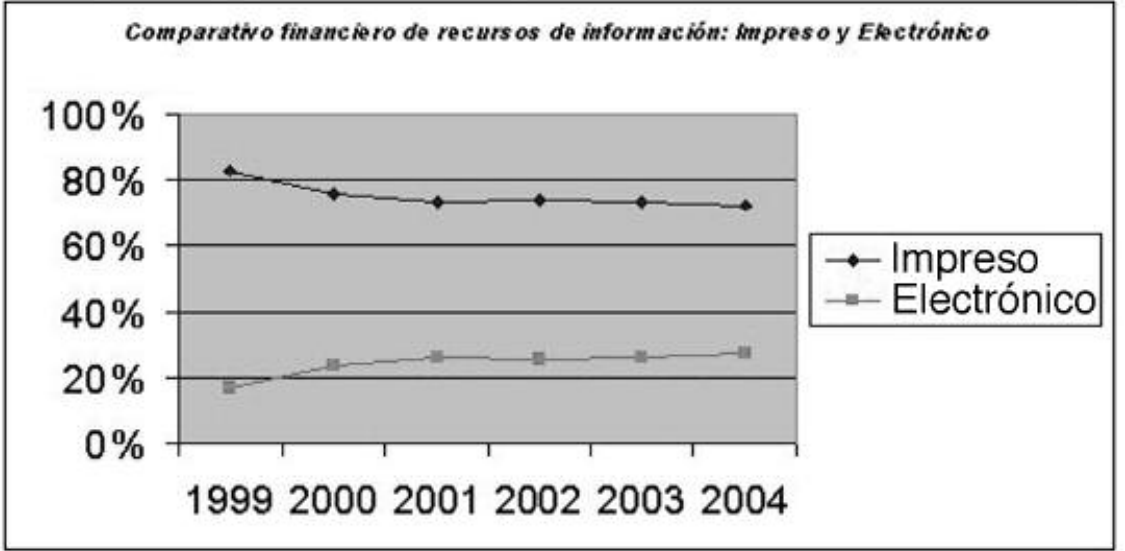

Figura 1 electrónicos de los cuales se tenga suscripción en papel.

Para tomar decisiones respecto a la suscripción de recursos de información, se creó en 2002 el Comité Evaluador de Bases de Datos y Recursos Electrónicos cuya misión es «conocer, evaluar y recomendar la renovación, cancelación, o nueva suscripción de recursos de información digital, con el propósito de optimizar los bienes económicos de la UNAM así como asegurar que la información digital se consulte y se explote adecuadamente"

En este contexto, en el año 2003, se creó un subcomité de racionalización de los recursos asignados a revistas técnicas y científicas, cuyo objetivo es el "de evaluar las colecciones de publicaciones periódicas actuales con el fin de optimizar los recursos informativos haciendo una redistribución del presupuesto destinado a la adquisición de la revista técnica y científica"12.

El subcomité propuso el Proyecto de racionalización de recursos asignados a revistas científicas y técnicas de la UNAM, cuyo objetivo fue "evaluar las colecciones de

\begin{tabular}{|c|c|c|c|c|c|c|c|}
\hline Suscripciones & 1998 & 1999 & 2000 & 2001 & 2002 & 2003 & 2004 \\
\hline $\begin{array}{c}\text { Títulos únicos de } \\
\text { revistas en papel }\end{array}$ & 7.931 & 8.000 & 8.000 & 8.000 & 8.000 & 7.570 \\
\hline $\begin{array}{c}\text { Total de revistas en } \\
\text { papel (incluye: } \\
\text { duplicados, } \\
\text { triplicados, etc.) }\end{array}$ & 13.885 & 13.516 & 13.500 & 13.500 & 13.500 & 12.934 \\
\hline Revistas electrónicas & 3.149 & & 6.000 & 6.073 & 6.400 & 7.300 & 7.337 \\
\hline
\end{tabular}

Tabla 2

publicaciones periódicas actuales con el fin de optimizar los recursos informativos haciendo una redistribución del presupuesto centralizado en la partida presupuestal 523 destinado a la adquisición de revistas técnicas y científicas, de acuerdo con la demanda de la comunidad académica.» ${ }^{13}$.

Este proyecto involucró: 265 títulos, 524 suscripciones duplicadas en papel correspondientes a 23 bibliotecas de facultades y escuelas, así como a 34 bibliotecas de la coordinación de la investigación científica, de las cuales por lo menos 25 se propusieron como bibliotecas depositarias $^{14}$. El resultado entre 2003 y 2004 fue la cancelación de 270 suscripciones con un ahorro de 282.686 US\$. Actualmente este proyecto sigue vigente con el firme objetivo de cancelar duplicados y redistribuir el ahorro de estás cancelaciones en la adquisición de nuevos títulos de revistas.

Ahora bien, se sabe que el proyecto de racionalización de recursos sólo puede asumir parcialmente la redistribución del número de suscripciones contratadas, pues en última instancia cada biblioteca decide los títulos que cancela. Por otra parte, la opción de cancelar revistas en cualquier formato no resuelve en sí la crisis de las revistas, ya que mientras en las bibliotecas se trabaja para identificar los títulos duplicados y los no utilizados para cancelarlos, los editores y proveedores 
más importantes mantienen sus políticas de edición y distribución, y de precios en constante incremento.

Por ello la cancelación de revistas libera pocos fondos, insuficientes para la adquisición de las nuevas suscripciones que la comunidad solicita, ya sea por tratarse de nuevas publicaciones o debido a líneas de investigación emergentes que demandan nuevos títulos.

En la tabla 2 exponemos una comparativa de revistas suscritas por la $D G B$ para todo el sistema bibliotecario, entre 1998 y $2004^{15}$. Puede notarse que hubo una reducción total de 361 títulos únicos suscritos en papel, lo que representa el $4,6 \%$ y de 951 del total de revistas en papel, que equivale al 6,9\%; de éstas, las suscripciones canceladas como parte del proyecto de racionalización fueron 270. Por otra parte se observa un incremento de 4.188 revistas electrónicas en el período expuesto, es decir, el 133\%, lo cual no implica tener más títulos, pues como antes se expuso es política de la $D G B$ adquirir en formato electrónico solamente aquellos que se tengan en papel.

La $D G B$ emite sus propias políticas de adquisición centralizada que son acatadas por las 142 bibliotecas que integran la red universitaria, y tienen la finalidad de agilizar los procedimientos y aminorar los tiempos en la adquisición de las fuentes electrónicas de información. Sin embargo en ocasiones entorpecen los procedimientos y la adquisición es complicada y sumamente lenta. Este hecho repercute y afecta a todos los usuarios potenciales de la universidad, en especial a los de centros e institutos de investigación.

\section{¿Cómo actúa la DGB ante esta problemática?}

Se ha iniciado un proceso de cooperación para la adquisición de revistas. Constituye una especie de consorcio -que no lo es en el término más estricto de la palabra- de políticas comunes respecto a la diversidad de licencias de acceso y a modelos de precios de la información electrónica. Estas políticas contemplan las condiciones de coste, uso, almacenamiento y acceso.

Para poder llevar a cabo la adquisición cooperativa se cita a continuación una serie de responsabilidades y acciones de la propia $D G B$ $\mathrm{y}$ de las bibliotecas que integran la red $^{16}$ :

_Las 142 bibliotecas de la UNAM proporcionarán todos los registros de las publicaciones periódicas suscritas, así como el archivo de todo su acervo para alimentar al catálogo colectivo de la $D G B^{17}$.

-Conjuntando todos los listados del sistema bibliotecario, se podrá conformar una lista general de revistas por disciplinas. $\mathrm{Si}$ dos o más bibliotecas tuvieran alguno interés en común, podrán realizar adquisiciones cooperativas y compartir recursos electrónicos.

-La Subdirección de Servicios de Información Especializada de la $D G B$, realizará estudios de evaluación de indicadores de uso de las revistas electrónicas, estudios comparativos entre proveedores de recursos de información y análisis de costo beneficio de las publicaciones, ya que el objetivo real es conocer su utilidad para la comunidad universitaria.

— Todos los centros deberán realizar estudios bibliométricos pertinentes por disciplina; los resultados (listados de títulos) serán la base para poder decidir la cancelación o renovación de la suscripción de determinadas publicaciones.

- Siempre se prestará el servicio interbibliotecario.

-Tendrán preferencia aquellas bibliotecas depositarias de los títulos únicos que tengan la colección más completa y se comprometerán a resguardar y mantener la suscripción, a proporcionar información sobre los títulos que preserven y a contar con recursos tecnológicos y humanos suficientes para albergar los títulos únicos.

- Se suscribirá el Programa de suministro de documentos, que tiene como propósito intercambiar documentos de manera eficaz y oportuna con el software Ariel que se instalará en todas las bibliotecas.

Estas medidas son todavía insuficientes para resolver el problema, por lo que es necesario observar las acciones de otras bibliotecas para optimizar el uso de sus recursos, así como negociar mejores acuerdos con los proveedores de información.

En 1997 un grupo de bibliotecas universitarias de Alemania y Holanda establecieron principios generales para las condiciones de acceso a revistas electrónicas y acuerdos de licencias con editores ${ }^{18}$. Esta propuesta fue muy bien acogida por la comunidad bibliotecaria en general, por lo que diversas asociaciones internacionales han logrado acuerdos en este sentido, por ejemplo la Ligue des bibliothèques européennes de recherche (Liber) o la International coalition of library consortia $(\text { Icolc })^{19}$.

En términos generales, los principales elementos de estos acuerdos son:

- Necesidad de cooperación entre editores y bibliotecas.

-Obtención de estadísticas de uso según estándares establecidos.

-Uso a perpetuidad de los archivos electrónicos y garantías de almacenamiento.

-Apoyo al préstamo interbibliotecario en papel.

-Acceso simultáneo al formato electrónico.

-Uso justo y razonable de la información contratada. 


\section{Años de experiencia}

Esto es lo que EBSCO ofrece.

Nuestro personal gestiona sus suscripciones a revistas electrónicas individuales o incluidas en ' paquetes de revistas, suscripciones, en papel y bases de datoś.

La Lista A-Z (A-to-Z) agrupa todos: sus recursos electrónicos en una misma lista, para que sus usuariọs localicen de manera rápida los títulos disponibles y accedan fácilmente a los contenidos. Además, la lista A-to-Z alimenta ál servidor de enlaces LinkSource ${ }^{\text {, }}$, que utilizando la norma Open'URL enlaza todos sus recursos de forma compacta e inteligente.

Experiencia, servicio, contenido, soluciones.

Hablemos hoy de sus necesidades.

CUSTOMERFOCUSEDCONTENTDRIVEN

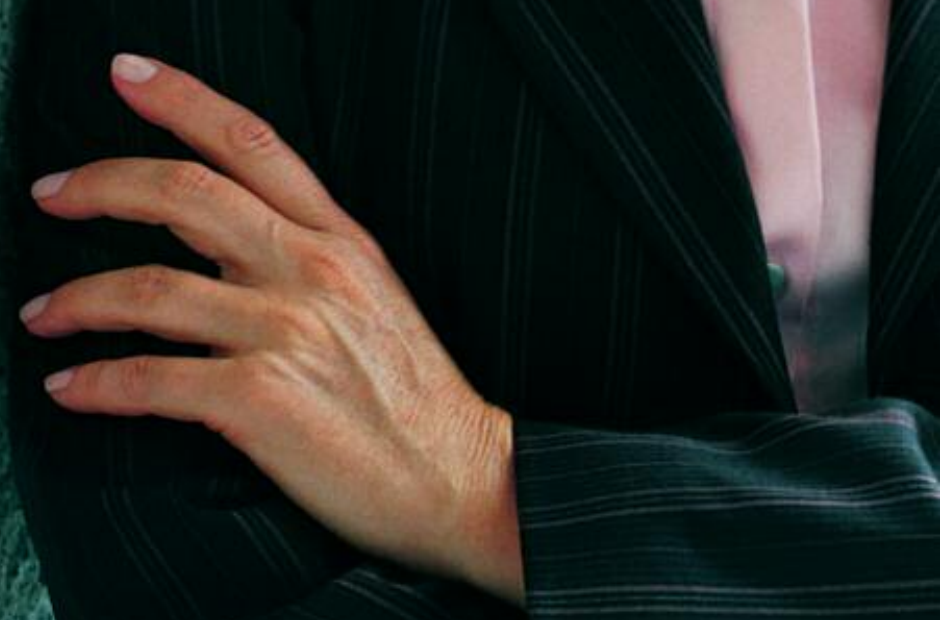
www.ebsco.com
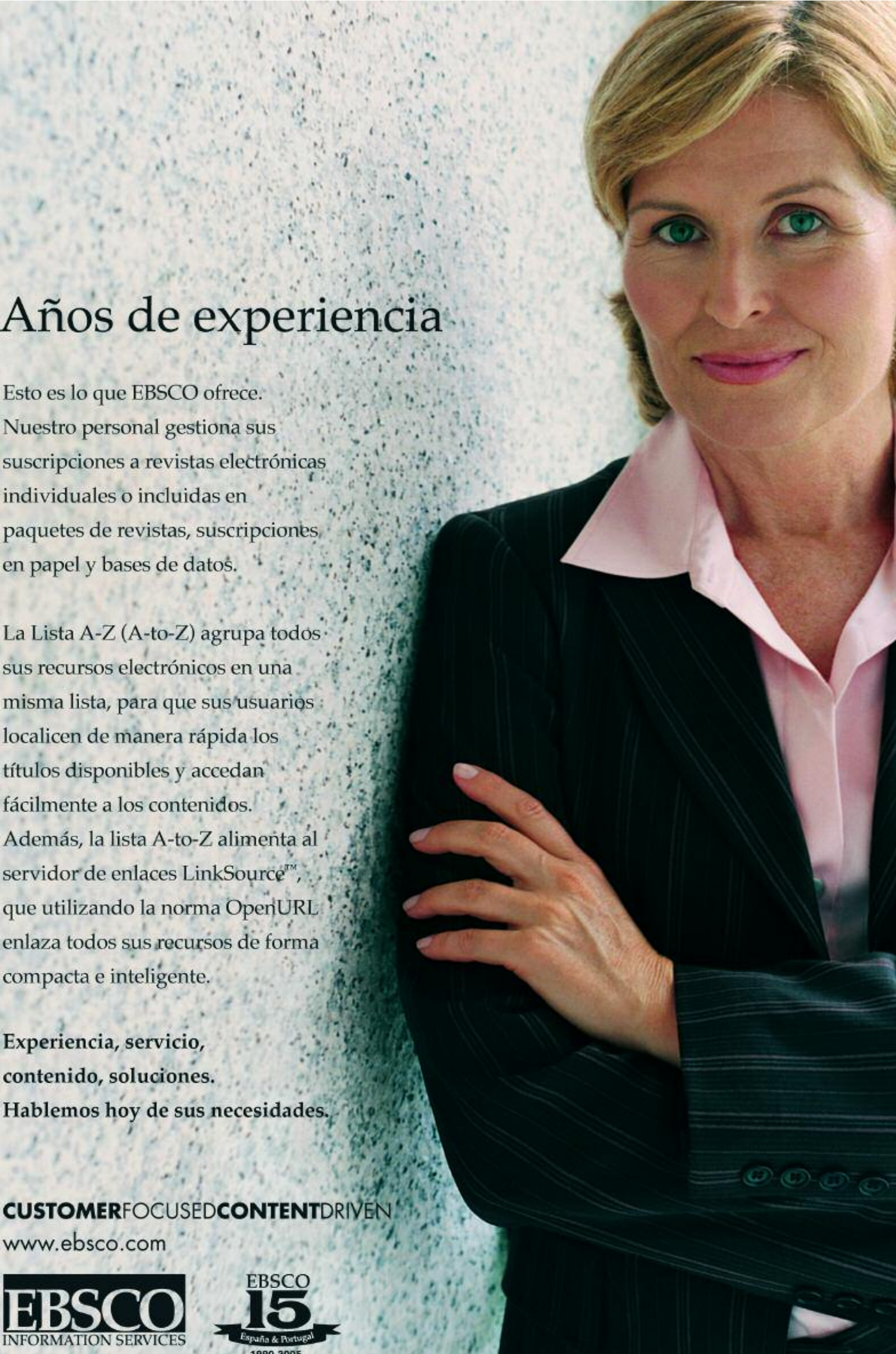
- Coste adicional para el formato electrónico que no exceda de un determinado porcentaje.

-Utilización de estándares para la integración de datos en los sistemas locales.

- Al contratar solamente suscripción electrónica, costos inferiores a los del formato en papel.

Este tipo de principios en la negociación de las licencias de acceso y adquisición de recursos de información electrónica pueden proporcionar a las bibliotecas de UNAM un punto de partida para obtener mejores condiciones de negociación con los proveedores y editores de revistas. Además, la UNAM podría establecer una postura y estrategia conjunta con otras bibliotecas universitarias de América Latina para procurar nuevos acuerdos en este mercado emergente.

La $U N A M$ no puede mantenerse ajena a las diferentes iniciativas que en el campo de la edición electrónica se están llevando a cabo, bien como acciones particulares o como fruto de actividades de asociaciones internacionales como las antes expuestas, o las de Sparc ${ }^{20}$-Scholarly Publishing and Academia Resources Coalition de la ARL ${ }^{21}$ (Association of Research Libraries)-, cuyos objetivos en colaboración con editores son:

- Crear un mercado más competitivo con objetivo de reducir el coste de adquisición de las revistas, impulsando publicaciones electrónicas de calidad.

-Asegurar el uso de los recursos electrónicos, fortaleciendo al mismo tiempo los derechos de los autores

-Aplicar tecnologías para mejorar los procesos de comunicación universitaria y académica, reduciendo los costes de producción y distribución.
De cara al futuro y de acuerdo con lo antes expuesto se propone:

1. La creación de un comité técnico encargado de fijar las políticas y la metodología de adquisición cooperativa de recursos de información, así como de realizar el seguimiento y coordinar el proyecto, que a su vez esté conformado por profesionistas relacionados con el área del conocimiento de cada centro, instituto, facultad o escuela de la UNAM para quien se destinen los recursos.

2. La redacción de un reglamento de aplicación en las bibliotecas de la UNAM que establezca principios a seguir en la selección y adquisición de productos de información electrónica, que adapte las pautas y principios internacionales a la situación mexicana.

3. Impulsar experiencias de edición electrónica de revistas producidas por la $U N A M$, así como portales de acceso a información de interés para determinadas comunidades científicas.

4. Integración de un equipo de profesionales multidisciplinario para la realización de estudios bibliométricos que sirvan como indicadores para la toma de decisiones.

\section{Notas}

1. Apud. Iñigo Marauri, El país, 27 de enero de 2004.

2. Lugo Hubp, Margarita; Orozco Aguirre, Aurelia. "La revista electrónica en la UNAM: retos presentes y futuros". En: Biblioteca universitaria, nueva época, julio-agosto, 2003, v. 6, n. 2, p. 141. Fuente: Departamento de Suscripciones, 2003.

3. Ibid. (los datos de 2004, fueron un estimado que hicieron las autoras).

4. Apud. Kyrillidou, Martha; Young, Mark. ARL library trends.

http://www.arl.org/stats/arlstat/04pub/04intro.html

5. Lugo Hubp, Margarita; Orozco Aguirre, Aurelia, op. cit., p. 141.

\section{Boletín estadístico de la UNAM, 2004}

7. «The journals crisis and the University of Adelaide». En: The University of Adelaide new library, 1999, n. 18.

http://www.library.adelaide.edu.au/ual/publ/new s/NEWS_18
8. Scholarly journals in crisis. The library connection-news from the Syracuse University Library.

http://libwww.syr.edu/information/libassoc/conn ection/fall99.pdf

9. Los coordinadores de bibliotecas nos han hecho saber que en general sus usuarios se resisten a que se cancelen las revistas en papel, aun teniendo los títulos en formato electrónico. Por otra parte, de todos es sabido que el formato electrónico, a pesar de todas sus ventajas, aún no garantiza su conservación en los términos que sí lo hace el soporte en papel.

10. Por ejemplo, Iñigo Marauri (op. cit.): “el notable aumento en el precio de las revistas relacionadas con las ciencias y la tecnología en los últimos años, con encarecimientos en algunos casos del 300\%".

11. http://www.dgbiblio.unam.mx/servicios/ gestionacceso.htm

12. (1) op. cit. 144.

13. Documento interno de la Subdirección de Servicios Especializados.

14. (2) op. cit., p. 146. Los requisitos que se consideraron para designar a las bibliotecas depositarias son: a) Que sea preferentemente la que tenga la colección más completa. b) Que se comprometa por escrito el titular de la dependencia y la $D G B$ a mantener la suscripción mientras se siga publicando el título y éste siga siendo relevante para la comunidad. c) Que proporcione los títulos solicitados por otras bibliotecas en caso de problemas con la versión electrónica. Para ello, debe contar con la infraestructura tecnológica necesaria y el personal profesional capacitado, para brindar un buen programa de suministro de documentos. d) Que disponga de los recursos humanos, de equipo y espacio para poder albergar los títulos en custodia.

15. Memoria-UNAM 1998, 2000, 2001, 2002, 2003, 2004.

16. Apud. En distintos documentos internos de la $D G B$.

17. El catálogo se llama Seriunam y puede consultarse en la web.

http://dgb.unam.mx

18. Licensing principles of the Dutch and German University Libraries.

http://webdoc.sub.gwdg.de/ebook/aw/prinzliz/1_ lizp-e.htm

19. Icolc statement of current perspective and preferred practices for the selection and purchase of electronic information.

http://www.library.yale.edu/consortia/statement. html

20. http://www.arl.org/sparc/about/index.html

21. http://www.arl.org/

María Esther Ramírez Godoy, Alma

Silvia Díaz, Escoto, Universidad Nacional Autónoma de México, Dirección General de Bibliotecas, Subdirección de Servicios Especializados. eramirez@dgb.unam.mx sdiaz@dgb.unam.mx 